\title{
Cadre réglementaire pour les médicaments dérivés du sang. Utilisation en Odontologie et en Chirurgie Buccale Etude descriptive de 46 interventions sur la période 2003 à 2004
}

\section{Regulation scope of blood derived product. Use in dentistry and in oral surgery Descriptive survey of 46 cases from 2003 to 2004}

\author{
J ULIEN GARNIER *, XAVIER FRACHON*, XAVIER MÉZIÈRE*, FABRICE CLIPET*, J ÉRÔME QUERO*,
} BENOÎT GUILLET **, PIERRE GUÉRET **, GILBERT DE MELLO *

\section{RÉSUMÉ}

L'objectif de ce travail est de présenter les médicaments dérivés du sang (MDS), de leur origine à leur application clinique en odontologie et en chirurgie buccale.

Dans une première partie nous abordons le cadre réglementaire et la pharmacovigilance spécifique pour les MDS. La deuxième partie aborde la sécurité virale et les différents moyens de production des MDS. La dernière partie montre les applications des MDS en odontologie et en chirurgie buccale dans notre service, illustrées par une étude descriptive.

Nous retiendrons que la législation des MDS a bien évolué depuis 1993. (Med Buccale Chir Buccale 2005 ; 11 :145-58).

mots clés : cadre réglementaire, facteur antihémophilique, colle biologique, extraction dentaire, antiagrégant plaquettaire, anticoagulant

médecin buccale chirurgie buccale VOL. 11, N 2005 page 145

\section{SUMMARY}

The aim of this work is to present the blood derived products (BDP), from their origin to their clinical application in dentistry and in oral surgery.

In the first part we approach the regulation scope and the specific monitoring of the side effects of BDP. The second part deals with the viral safety and the production of BDP. The last part regards the applic ations of BDP in dentistry in our service, and is illustrated by a descriptive survey.

To conclude we have to remember that the legislation regarding BDP has evolved in France since 1993 and 1998, and is becoming homogenous in the European Community. The improvement of the techniques of purification and of the viral safety have made it possible to improve the patients care respecting the sanitary security. (Med Buccale Chir Buccale 2005 ; 11 : 145-58).

key words : regulation scope, antihemophilic factor, fib rine glue, tooth extraction, antiplatelet agent, antic oagulant

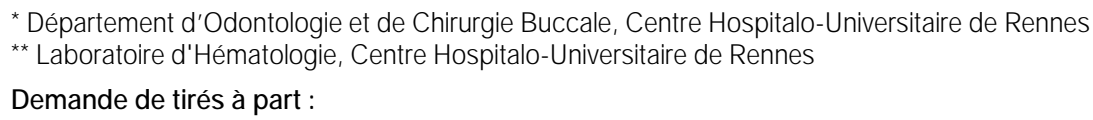


médecine buccale chirurgie buccale

L. $11, \mathrm{~N}^{\circ} 3$ 2005

page 146
Le plasma humain contient de nombreuses protéines, dont les protéines de la coagulation en concentration infime. Elles peuvent être extraites après plusieurs étapes de purification et être utilisées comme moyen thérapeutique. On parle de produits sanguins stables ou de médicaments dérivés du sang (MDS). Les MDS ont été à l'origine de contamination massive par le virus de l'hépatite $C$ (VHC) de la fin des années 1970 jusqu'au début des années 1990, et par le virus de l'immunodéficience humaine $(\mathrm{VIH})$ jusque dans les années 1984/85. Depuis, la sélection rigoureuse des donneurs de sang a permis de diminuer très significativement l'incidence des lots de plasma contaminé. La transfusion de sang total ou de plasma a été le premier traitement de l'hémophilie jusqu'au milieu du XXe siècle. Le rendement de ces traitements était faible. En effet, les concentrations en facteurs VIII et IX étant infimes, il fallait transfuser des volumes importants de sang ou de plasma pour obtenir un effet hémostatique. De plus ces techniques nécessitaient une hospitalisation de 7 à 10 jours et les risques de contaminations virales étaient importants. Le PPSB (prothrombine, proconvertine, facteur de Stuart, facteur antihémophilique B) fut ensuite utilisé pour le traitement de I'hémo philie $B$. Mais les injections répétées et les doses élevées de PPSB pouvaient être responsables d'une coagulation intravasculaire disséminée. En 1964, P ool développa la cryoprécipitation comme technique de fractionnement du plasma [1] La congélation du plasma puis sa décongélation lente permettait d'obtenir un cryoprécipité riche en facteur VIII. Cette technique a considérablement amélioré la prise en charge des sujets hémophiles. Cependant, le cryoprécipité ne pouvait pas être assimilé à un véritable produit substitutif puisqu'il contenait d'autres protéines plasmatiques. II présentait par ailleurs un risque élevé de transmission virale. Le développement de nouvelles techniques de purification plasmatique (chromatographie, élimination/inactivation virale) ont permis la production de MDS plus concentrés, plus purs et de moins en moins contaminants. La pureté et les concentrations ainsi obtenues ont permis de diminuer le nombre et le volume des injections nécessaires, d'amélio rer la tolérance au traitement et d'augmenter la qualité de l'hémostase.
La loi 93-5 du 4 janvier 1993, relative à la sécurité en matière de transfusion sanguine et de médicament, distingue d'une part les produits sanguins labiles (éléments cellulaires) et d'autre part les produits sanguins stables, appelés MDS [2]. Cette loi donne le statut de médic ament à des produits sanguins d'origine humaine. Ils sont soumis à la législation encadrant les médicaments avec une pharmacovigilance renforcée. Le décret 95-566 du 6 mai 1995, relatif à la pharmacovigilance exercée sur les MDS humains impose la mise en place d'un système de traçabilité, parallèle à celui des produits sanguins labiles [3]. Ce système de traçabilité est un élément clé de la sécurité sanitaire car il permet le suivi des lots depuis le donneur jusqu'au receveur.

L'essor du génie génétique puis le clonage des gènes humains codant pour les facteurs VIII et IX ont permis la production de MDS d'origine recombinante. Ils ne sont plus obtenus à partir de plasma humain mais à partir de cellules en culture.

Les extractions dentaires entraînant bien souvent des hémorragies et de volumineux hématomes chez les patients ayant un trouble de l'hémostase, les MDS trouvent donc une indication de choix dans notre discipline, notamment les concentrés de facteurs antihémophiliques et la colle biologique.

\section{CADRE REGLEMENTAIRE}

Les bases du cadre réglementaire pour les MDS sont énoncées dans la loi $n^{\circ}$ 93-5 du 4 janvier 1993 relative à la sécurité en matière de transfusion sanguine et de médicament [2]. Cette loi fait suite à l'affaire du sang contaminé dans les années 1980 à 1985. A l'époque la sécurité transfusionnelle n'était pas aussi renforcée qu'aujourd'hui : la sélection des donneurs était moins rigoureuse et les tests biologiques sur les dons non systématiques. Elle a donc permis de renforcer la sécurité pour les MDS. Cette loi a permis de distinguer d'une part les produits sanguins labiles (PSL) et les MDS ; les premiers étant soumis à l'hémovigilance et les seconds à la pharmacovigilance puisqu'ils sont assimilés à des médicaments. La loi du 4 janvier 1993 [2] prévoit également la création de plu- 
sieurs organismes : l'agence du médicament (AM), l'agence française du sang (AFS), les établissements de transfusion sanguine (ETS), le comité de sécurité transfusionnelle (CST), le laboratoire français du fractionnement et des biotechnologies (LFB). Elle organise à travers ces différents organismes le don, la collecte du sang, la mise en place de règles éthiques, la qualification biologique des dons, la production des MDS et leur distribution.

En 1998, une nouvelle loi a été publiée au J ournal Officiel, il s'agit de la loi $n^{\circ} 98-535$ du $1^{\text {er juillet }}$ 1998 relative au renforcement de la veille sanitaire et du contrôle de la séc urité sanitaire des produits destinés à l'homme [4]. Elle paraît à l'époque où différentes affaires de santé publique rappellent l'affaire du sang contaminé : cancers dus à l'amiante, " poulets à la dioxine », maladie de la «vache folle». Comme le principe de précaution se généralise, cette nouvelle loi envisage la sécurité sanitaire au sens large, englobant les produits cosmétiques comme les couronnes dentaires, le maquillage en passant par les aliments et allant jusqu'aux MDS. Beaucoup plus générale que la précédente, elle prévoit la création de nouveaux organismes : le comité national de sécurité sanitaire, l'institut de veille sanitaire (IVS), l'agence française de sécurité sanitaire des produits de santé (AFSSAPS), l'établissement français du sang (EFS), l'agence française de sécurité sanitaire de l'environnement, l'agence française de sécurité sanitaire des aliments (AFSSA). Certains organismes créés par la loi de janvier 1993 sont regroupés pour former de nouvelles entités. L'AFS deviendra l'EFS, les ETS passeront sous le contrôle des EFS, l'agence du médicament sera remplacée par l'AFSSAPS (Fig. 1).

L'Union Européenne participe aussi à la mise en place du cadre réglementaire des MDS par des résolutions du Conseil de l'Union Européenne et des directives du Parlement Européen. La directive 89/381/CEE du Parlement Européen et du Conseil de l'Union Européenne du 14 juin 1989 prévoit des dispositions spéciales pour les MDS et les médicaments dérivés du sang ou du plasma humain [5]. Dans cette directive, les états membres prennent toutes les mesures utiles pour promou- voir l'autosuffisance de la communauté en sang et encouragent les dons de sang volontaires et non rémunérés. La résolution du Conseil de I'Union Européenne du 2 juin 1995 insiste sur la promotion du don volontaire, non rémunéré et sur la sécurité de la collecte du sang contribuant à la prévention des maladies transmissibles par le sang [6]. La directive 2002/98/CE du Parlement Européen et du Conseil de l'Union Européenne établit des normes de qualité et de sécurité pour la collecte, le contrôle, la transformation, la conservation et la distribution du sang humain et des composants sanguins [7]. L'objectif de ces textes est d'assurer un niveau élevé de protection pour la santé humaine.

Les MDS sont soumis à une pharmacovigilance stricte [3, 8-10]. La pharmacovigilance, ou surveillance des effets indésirables des médicaments ap rès leur mise sur le marché, s'avère particulièrement nécessaire pour ces produits. Lorsqu'un médicament est mis sur le marché, il a été validé par des essais cliniques de phases I, II et III. Les patients utilisant les molécules en cours d'expérimentation sont en général sélectionnés par des critères rigoureux d'inclusion et d'exclusion. De ce fait, le prescripteur et le fabricant n'ont pas suffisamment de recul et certains effets indésirables seront découverts ultérieurement. En ce qui concerne les MDS, le risque est majoré puisque le nombre de patients inclus dans les essais cliniques est faible. La pharmacovigilance permet d'identifier, d'évaluer et de prévenir le risque d'effets indésirables pour les médicaments commercialisés avec une autorisation de mise sur le marché (AM M) ou une autorisation temporaire d'utilisation (ATU).

Selon l'addendum de 1998 pour la pharmacopée européenne, les donneurs et les dons de sang doivent être enregistrés pour pouvoir retrouver l'origine d'un éventuel lot de MDS contaminant [11]. La pharmacovigilance qui s'applique à tous les médicaments comporte des règles supplémentaires spécifiques pour les MDS. La législation traitant la pharmacovigilance des MDS est contenue dans deux décrets : le décret n 95-278 du 13 mars 1995 relatif à la pharmacovigilance [8], le décret $n^{\circ} 95-566 \mathrm{du}$ 6 mai 1995 relatif à la pharmacovigilance exer- médecin€ buccale chirurgie buccale VOL. $11, \mathrm{~N}$ 2005 page 147 
médecine buccale chirurgie buccale

L. $11, \mathrm{~N}^{\circ} 3$ 2005 page 148

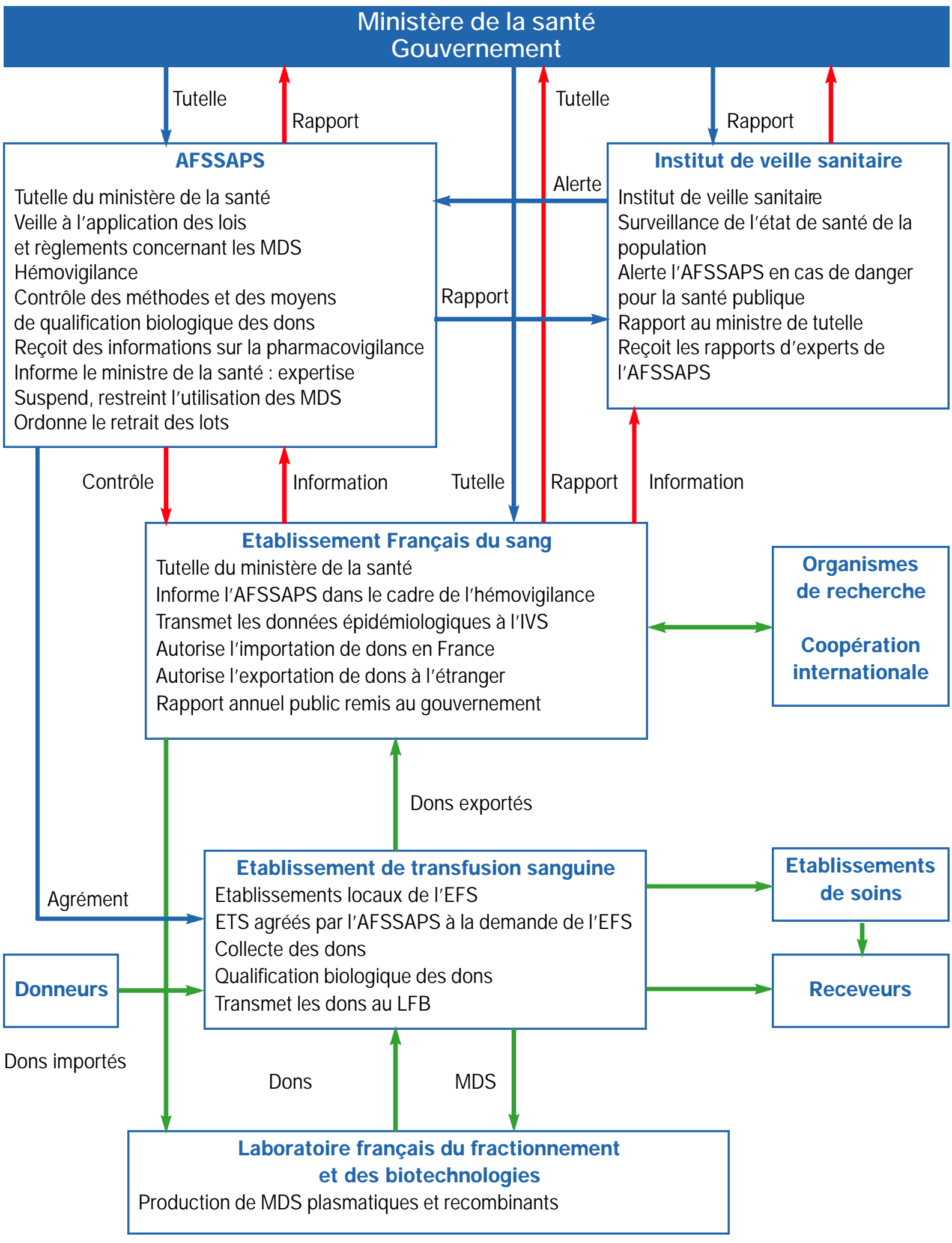

Figure 1 : Schéma des relations entre les différents organismes. Links between the different institutions. 
cée sur les MDS [3]. Pour mémoire, tout médecin, pharmacien, chirurgien-dentiste ou sagefemme ayant constaté un effet indésirable grave ou inattendu, susceptible d'être dû à un médicament ayant une AM M, doit en faire la déclaration immédiatement. Tout membre d'une profession de santé ayant fait la même constatation peut également la notifier. La déclaration est formulée sur l'imprimé prévu pour la déclaration d'incident de pharmacovigilance. Les MDS ont le statut de médicament mais la législation les encadrant est plus stricte. Depuis I'affaire du sang contaminé des années 1980/85, un système de pharmacovigilance spécifique a été mis en place [12-15]. Le décret $n^{\circ}$ 95-566 du 6 mai 1995 relatif à la pharmacovigilance pour les MDS précise ce nouveau cadre réglementaire [3]:

- Création de correspondants de pharmacovigilance pour sur les MDS : le correspondant est responsable de l'enregistrement et de la consenvation des informations relatives aux MDS. II reçoit et transmet au centre régional de pharmacovigilance les déclarations d'effets indésirables susceptibles d'être liés aux MDS qu'il a délivrés.

- Création d'un système de traçabilité : la traçabilité est spécifique aux MDS. Elle permet le suivi des molécules depuis le prélèvement sanguin jusqu'au patient recevant le médicament. Ce système permet l'identification et le suivi de tous les lots. Elle est définie par l'article R . 5144-25 du code de santé publique [3]. La traçabilité a permis un gain de sécurité mais aussi une meilleure utilisation et une meilleure gestion des MDS. Le suivi des MDS est possible par la mise en place d'un système de formalités depuis la production à l'administration. Cela concerne les établissements qui prélèvent le sang, ceux qui produisent les MDS, les distributeurs (pharmacies), les prescripteurs et les personnes qui administrent les MDS. A chaque étape, il y a identification du numéro des lots.

- Mise en place de l'obligation de signalement: l'objectif principal de ce système est de prévenir la multiplication des incidents transfusionnels. Chaque incident signalé est répertorié et mis en relation avec le prélèvement d'origine. Ceci permet d'écarter tous les MDS fabriqués à partir du même lot contaminé.

\section{PRODUCTION DES MDS}

Les MDS sont d'origine plasmatique ou recombinante. Les deux principales procédures intervenant dans la production des MDS d'origine plasmatique sont le fractionnement plasmatique et l'élimination/inactivation virale. L'objectif de ces techniques est d'obtenir le produit le plus pur possible, sans altération des propriétés physicochimiques et biologiques de la protéine d'intérêt et sans aucun agent infectieux contaminant. Selon l'addendum de 1998 pour la pharmacopée européenne [11], le plasma humain pour le fractionnement correspond à la fraction liquide du sang humain recueillie sur un antico agulant, séparée des éléments figurés du sang, par filtration continue ou par centrifugation (aphérèse) [16-19]. Les MDS d'origine plasmatique sont obtenus par des techniques qui permettent la séparation, dans les pools plasmatiques recueillis, des différentes protéines plasmatiques humaines et l'élimination ou l'inactivation des agents infectieux transmissibles. L'albumine est la principale protéine du plasma,

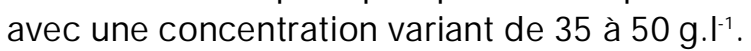
Les facteurs de coagulation sont présents dans des concentrations nettement plus faibles, de 0,1 à $0,2 \mathrm{mg} . \mathrm{I}^{-1}$ pour le facteur VIII et de 3 à $7 \mathrm{mg} \cdot \mathrm{I}^{-1}$ pour le facteur IX (Fig. 2).

\begin{tabular}{|lr|}
\hline Albumine & $35000-50000$ \\
IgG & $8000-18000$ \\
Fibrinogène & $2000-4500$ \\
Alpha-1-antitrypsine & $1300-3000$ \\
IgA & $900-4500$ \\
IgM & $600-2500$ \\
Antithrombine III & $220-390$ \\
Transferrine & $200-320$ \\
Alpha-2-macroglobuline & $150-420$ \\
Haptoglobine & $150-300$ \\
Inter-alpha-trypsine-inhibiteur & $20-70$ \\
Facteur Willebrand & $4-6$ \\
Facteur IX & $3-7$ \\
Protéine C & $3-7$ \\
Facteur VIII & $0,1-0,2$ \\
\hline
\end{tabular}

Figure 2 : Composition protéique du plasma (en $\mathrm{mg} \cdot \mathrm{I}^{-1}$ ) Protein concentration in plasma $\left(\mathrm{mg} . \mathrm{I}^{-1}\right)^{[20]}$ médecin€ buccale chirurgie buccale VOL. $11, \mathrm{~N}$ 2005 page 149 
Les pools (appelés « lots de plasma ») sont réalisés par le mélange de plusieurs milliers de dons (appelés «unités plasmatiques ») (5000 à 15 000poches de plasma). Ce procédé augmente la probabilité de contaminer un lot entier. En effet, si un virus est présent dans une seule unité plasmatique, il contaminera l'ensemble du lot. C'est une des raisons principales de la contamination antérieure des hémophiles par le VIH, le VHB et le VHC. C'est la raison pour laquelle les plasmas recueillis subissent trois étapes successives de contrôle antivirale avant purification sélective de la protéine recherchée:

1) Contrôle qualité des unités de plasma :

- anticorps anti-VIH 1 et anti-VIH 2,

- antigène HBs (antigène de surface du VHB),

- anticorps anti-VHC,

- recherche du génome du parvovirus B19, du VHA et du VHC par polymerase chain reaction (PCR).

2) Phase de quarantaine de 90 jours minimum avant fractionnement.

3) Contrôle des lots de plasma avant fractionnement :

- anticorps anti-VIH 1 et anti-VIH 2,

- antigène HBs (antigène de surface du VHB),

- anticorps anti-VHC,

- recherche du génome des virus $P$ arvovirus B19, du VHA, du VHB, du VHC et du VIH par polymerase chain reaction (PCR) ou «PCR 5 virus».

L'efficacité de l'élimination/inactivation virale reste limitée vis à vis de certains virus non enveloppés résistants (Parvovirus B19 et VHA). C'est pourquoi des techniques d'amplific ation génomiques pour certains de ces virus sont mises en œuvre dès la réception du plasma. Un test positif entraîne la destruction de la poche.

\section{Procédures de purification des MDS}

Le fractionnement du plasma associe une technique de précipitation, permettant une séparation des principales protéines plasmatiques à une technique de chromatographie permettant une sélection des protéines plasmatiques présentes en faible concentration [20,21]. Ces moyens doivent respecter certains critères :

- obtenir un produit thérapeutique efficace,
- permettre une valorisation optimale des dons de plasma en purifiant toutes les protéines plasmatiques thérapeutiques.

La principale technique de précipitation est la cryoprécipitation. Le plasma humain recueilli pour le fractionnement est conservé à une température inférieure ou égale à $-20^{\circ} \mathrm{C}$. On obtient une cryoprécipitation en décongelant le plasma à une température comprise entre +2 et $+4^{\circ} \mathrm{C}$; il en résulte un cryosurnageant et un cryoprécipité qui seront séparés par centrifugation ( $\mathrm{Fig}$. 3). P ool montra en 1964 l'intérêt du cryoprécipité et notamment leur richesse en facteurs antihémophiliques par rapport au plasma frais qui était utilisé auparavant [1]. Ce fut un tournant dans la prise en charge des patients hémophiles. Le cryoprécipité a longtemps servi au traitement de l'hémophilie A et de la maladie de Willebrand, étant donné sa composition [21]. Néanmoins son utilisation comporte des limites infranchissables:

- elle ne permet pas l'inactivation virale et doit donc être suivie de méthodes d'inactivation/élimination virale ;

- le fractionnement doit être complété par des chromatographies d'affinité pour améliorer la purification ;

- l'utilisation du cryoprécipité seul, sans purification supplémentaire, est contre productif et ne permet pas une valorisation suffisante des dons de sang [20].

Les techniques chromatographiques utilisées dans le fractionnement plasmatique permettent une sélection fine et une purification des différentes protéines plasmatiques, surtout de celles présentes en faible concentration. Elles ne doivent pas dénaturer la structure des protéines [20,22], ce qui pourrait les rendre non fonctionnelles et/ou plus immunogènes. Elles peuvent être utilisées avec différents objectifs :

- purifier sélectivement les protéines d'intérêt,

- séparer les polymères des monomères,

- éliminer des stabilisants, des agents virucides et des sels,

- participer à la réduction virale.

Les principales méthodes chromatographiques sont:

- l'échange d'ions (anions ou cations),

- l'immuno-affinité,

- l'exclusion stérique. 


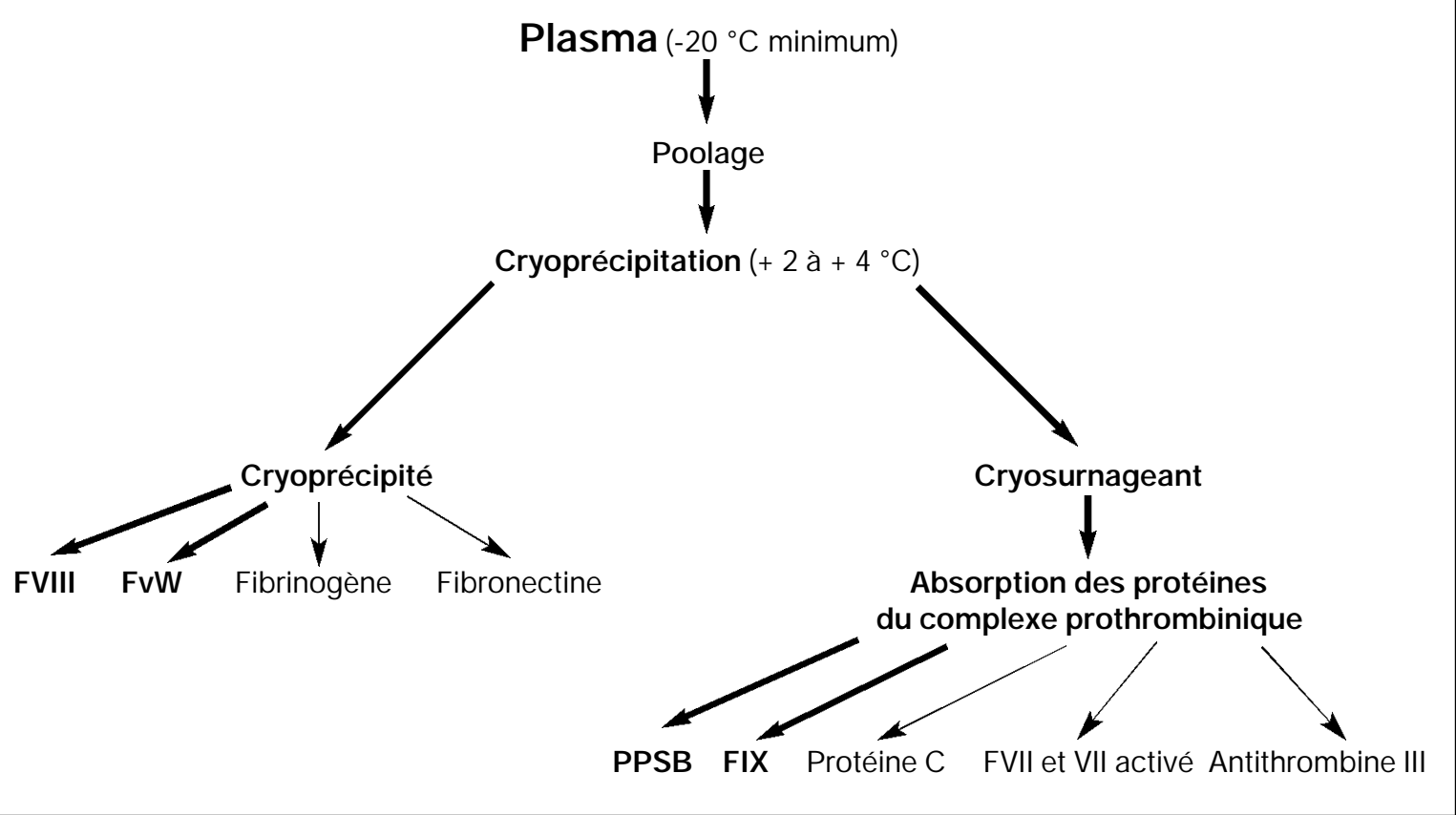

Figure 3 : Principe du fractionnement plasmatique par cryoprécipitation Fractionation of plasma by cryoprecipitation

\section{Sécurité virale des MDS d'origine plas- matique}

Des mesures de sécurité virale sont prises depuis le don du sang (sélection des donneurs, tests et analyses de dépistage, qualification du don...) jusqu'au fractionnement du plasma [9]. Malgré tout il peut persister des risques de contamination virale des MDS. Pour cette raison, des procédés d'élimination/inactivation virale sont directement intégrés dans la production des MDS. Ces méthodes constituent la dernière étape de la sécurité virale.

L'objectif des méthodes d'élimination/inactivation virale est d'assurer l'inactivation d'au moins 4 ou $5 \log$ (10 000 à 100000 particules infectieuses par $\mathrm{ml}$ de solution protéique) des $\mathrm{VIH} 1$ et 2, du VHB, du VHC et du parvovirus B19, tout en préservant l'activité biologique des protéines. Les principales techniques sont d'ordre :

- physique (par exemple la pasteurisation),

- chimique (par exemple les solvants/détergents),

- par nanofiltration (par exemple des filtres avec pores de $15 \mathrm{~nm}$ pour le FIX).
Les MDS d'origine recombinante [21]

page 151
Ils sont produits grâce au génie génétique et ils ne font pas appel au don de sang. La production de ces médicaments a été possible par le clonage des gènes codants pour les facteurs VII, VIII et IX. Des protéines de structure primaire identique à celle des protéines naturelles sont produites par des cellules d'origine animale en culture dans lesquelles a été inséré l'ADN complémentaire (ADN sans les introns) de la protéine recherchée. Ces médic aments ont le même effet thérapeutique que les protéines d'origine plasmatique [23]. Les MDS d'origine recombinante ne remplacent pas toutes les protéines plasmatiques. Les MDS d'origine recombinante comporte des avantages :

- leur disponibilité n'est pas fonction du volume des dons de sang, ce qui diminue le risque de situation de pénurie mais ne l'exclut pas ;

- la transmission des virus d'origine humaine est impossible ;

- ils ont une très haute pureté.

L'inconvénient principal est la production d'anticorps anti-facteur de la coagulation. Des études 
médecine buccale chirurgie buccale sont menées sur des groupes de patients hémophiles PUPs (Previously Untreated Patients) n'ayant jamais reçu de facteurs de coagulation d'origine plasmatique. D'après Bray et coll. $23,9 \%$ des PUPs traités par du Recombinate ${ }^{\circledR}$ (FVIII recombinant) développent des anticorps antifacteur VIII [24]. Avec le Kogénate ${ }^{\circledR}$ (FVIII recomb inant), les anticorps apparaissent entre 1 mois et 5 ans après le début du traitement; les 2/3 apparaissent pendant la troisième année [23]. Chez I'hémophile $A$, le risque de développer des anticorps anti-FVIII lié à l'utilisation de concentrés recombinants ou plasmatiques, n'est pas prévisible mais on sait que les allo-anticorps apparaissent plus tôt avec les produits recombinants.

II existe 6 concentrés de facteurs antihémophiliques d'origine recombinante:

- Facteurs VIII recombinants :

- Recombinate ${ }^{\circledR}$ en cours de remplacement par l'Advate (sans albumine)

- Refacto ${ }^{\circledR}$

- Kogenate-Bayer ${ }^{\circledR}$

- Helixate Nexgen ${ }^{\circledR}$

- Facteur IX recombinant:

- Benefix ${ }^{\circledR}$

- Facteur VII activé recombinant (rFVIIa) :

- Novoseven ${ }^{\circledR}$

\section{Principes de la production des MDS d'origine recombinante [25-27]}

Les gènes humains codant pour les protéines des facteurs VII, VIII et IX ont été isolés et clonés dans le but de les introduire dans un vecteur d'ADN (vecteur plasmidique). Ce vecteur est ensuite transfecté dans des cellules de mammifères en culture bien caractérisées (lignées cellulaires). Elles assurent les modifications post-traductionnelles nécessaires à l'ac tivité coagulante des protéines. Ces lignées cellulaires modifiées sécréteront la protéine recherchée qui sera par la suite recueillie, puis purifiée. Ces lignées cellulaires sont:

- des cellules d'ovaires de hamster chinois (chinese hamster ovary ou $\mathrm{CHO}$ ),

- des cellules de reins de hamster nouveau-né (baby hamster kidney ou BHK).
La purification des protéines anti-hémophiliques recombinantes est réalisée par chromatographie d'immuno-affinité sur des colonnes d'anticorps monoclonaux spécifiques de la protéine recherchée. Cette dernière est spécifiquement retenue avec élimination du reste du milieu de culture cellulaire (Fig. 4) [9].

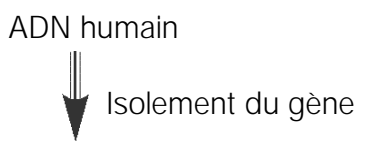

Gène codant pour le FAH (facteur antihémophilique)
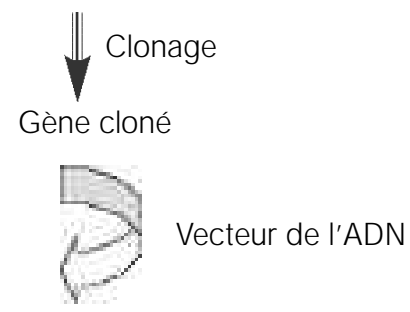

Lignées cellulaires hôtes $\mathrm{CHO}$ ou BHK

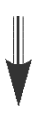

Milieu de culture<smiles>C1=CCCC1</smiles>

Production de FAH

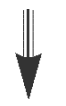

Purification et séparation du FAH

Chromatographie

Elimination/inactivation virale

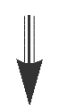

FAH stable dans son milieu de conservation

Figure 4 : Production des MDS recombinants [21] Production of recombinant BDP [21]

\section{UTILISATION DES MDS EN ODONTOLOGIE ET EN CHIRURGIE BUCCALE}

L'équipe de Samson et Bernard [28, 29] à Genève est l'une des premières à avoir décrit l'utilisation de la colle biologique en chirurgie buccale. $\mathrm{Ce}$ 
sont principalement les facteurs antihémophiliques et la colle biologique, composée de thrombine et fibrinogène. Ils permettent la réalisation d'actes chirurgicaux, en général des extractions dentaires, chez des patients ayant des troubles de I'hémostase. II s'agit principalement de patients ayant une maladie hémorragique héréditaire (hémophiles... ) ou de patients traités par antivitamine K (AVK), fortement anticoagulés, avec un INR supérieur ou égal à 2,8. Les extractions dentaires chez les patients fortement anticoagulés (INR $\geq 2,8)$ nécessite classiquement un relais avec une héparine de bas poids moléculaire (HBPM), permettant momentanément l'arrêt des $A V K$, tout en conservant une anticoagulation. Avec la colle biologique, on peut réaliser des interventions avec un INR supérieur ou égal à 2, 8 sans faire de relais à une HPBM. Cette colle est également utilisée pour les extractions en terrain irradié. Nous avons utilisé le registre des patients ayant un risque hémorragique de notre service pour analyser I'utilisation des MDS [30, 31]. Cette étude a pour but de montrer que l'utilisation des MDS dans notre discipline a permis une amélioration de la prise en charge des patients porteurs de troubles majeurs de l'hémostase et des patients irradiés. On a inclus dans cette étude rétrospective portant sur 12 mois - juin 2003 à juin 2004 tous les patients chez qui un MDS a été utilisé.

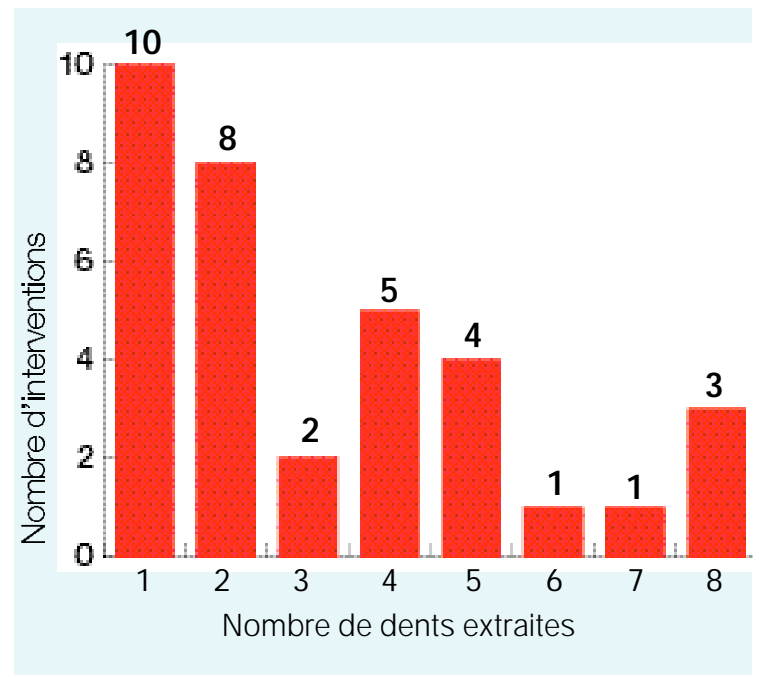

Figure 5 : Nombre de dents extraites par intervention Tooth extracted during each intervention
35 patients ( 6 femmes, 29 hommes) ont été traités avec des MDS. La majorité d'entre eux n'ont subi qu'une intervention, quatre ont été opérés deux fois, une patiente a été traitée quatre fois et une patiente cinq fois, pour un total de 46 interventions. Pour le détail, ces 46 interventions correspond ent à 142 extractions (Fig. 5), 8 curetages d'ostéite, 2 exérèses de kyste et 1 frénectomie. On distingue deux groupes principaux de patients : les patients ayant un trouble de l'hémostase et les patients ayant subi des interventions en territoire irradié (Fig. 6).

Le groupe de patients ayant un trouble de l'hémostase se composait de :

- 9 patients hémophiles,

- 2 patients atteints d'une maladie de Willebrand - 12 patients traités par AVK,

- 3 patients traités par l'association Kardégic ${ }^{\circledR}$ et Plavix ${ }^{\circledR}$,

- 4 patients ayant d'autres traitements anticoagulants : Calciparine ${ }^{\circledR}$ seule, Kardégic ${ }^{\circledR}$ et Lovénox ${ }^{\circledR}$ associés. Ces quatre patients étaient âgés, grabataires ou handicapés, avec des mouvements incontrôlés de la mandibule et des tics de succion ne permettant pas d'assurer une bonne hémostase locale par compression. C'est I'association du risque hémorragique et du comportement inadéquat du patient qui a justifié l'utilisation de colle biologique (Fig. 7).

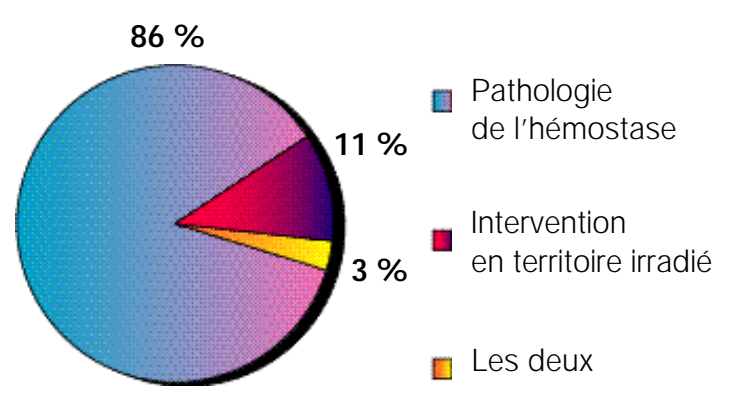

Figure 6 : Indications des MDS en odontologie et en chirurgie buccale Indication of BDP in dentistry and in oral surgery médecine buccale chirurgie buccale VOL. $11, \mathrm{~N}$ 2005 page 153 


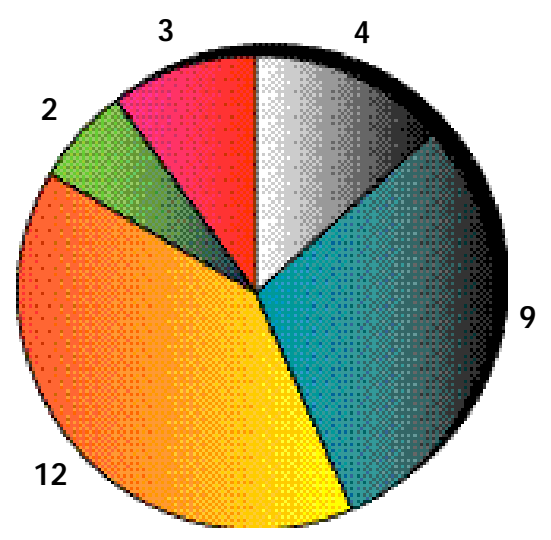

Patients hémophiles

Patients sous AVK

Maladies de Willebrand

Patients sous Kardégic ${ }^{\circledR}$ et Plavix ${ }^{\circledR}$

Autres

Figure 7 : Indications de la colle biologique dans les troubles de l'hémostase Indication of fibrin glue in haemostasis troubles

médecine buccale chirurgie buccale

L. $11, \mathrm{~N}^{\circ} 3$

2005

page 154
Dans le groupe des patients traités par AVK (Sintrom ${ }^{\circledR}$ ou Previscan ${ }^{\circledR}$ ), seuls les patients ayant un INR élevé (supérieur à 2,8) ont nécessité l'emploi de colle biologique [32, 33]. L'INR moyen était de 2,89 , le plus faible de 2,41 , le plus élevé de 3,85 . Toutes les interventions ont été réalisées en chirurgie ambulatoire, sans relais hép arinique, puisque dans tous les cas il s'agissait de patients à risque thrombo-embolique majeur, avec un INR difficile à stabiliser. Parmi ces patients deux ont bénéficié de exérèse d'un kyste, la colle biologique a servi à combler la cavité kystique. Aucun patient n'a eu de complications hémorragiques per ou post-opératoire. L'emploi de la colle biologique et des moyens d'hémostase locale ont permis de garantir une prise en charge simplifiée et une prévention thrombo-embolique efficace des patients traités par AVK [26].

Le groupe des patients hémophiles comportait 5 patients hémophiles $A$ mineurs, 1 hémophile $A$ modéré, 2 hémophiles $A$ sévères et 1 hémophile $B$ sévère ( $F$ ig. 8 ) [18, 27, 35-37]. Le patient hémophile $B$ sévère a subi 2 interventions, avec l'extraction de 8 dents sous anesthésie locale à chaque séance. Avant chaque interventions, 4000 unités de Betafact ${ }^{\circledR}$ ont été injectées. L'hémostase locale a été réalisée avec de la colle biologique, des plaques de collagène et des sutures, et le patient a été gardé sous surveillance pendant 24 heures. Pour les patients hémophiles $A$, les interventions ont été pratiquées en ambulatoire. Après contrôle du saignement en fin d'après-midi, le patient a été libéré. Aucun patient n'a néc essité la prolongation de son hospitalisation. Sur les 5 patients hémophiles $A$ mineurs, 1 seul a reçu une injection de Factane ${ }^{\circledR}$. Pour les 4 autres patients, I'hémostase locale a permis d'obtenir une hémostase suffisante. L'hémophile $A$ modéré et le premier hémophile sévère ont été traités parFactane ${ }^{\circledR}$. Le deuxième hémophile sévère était un enfant de huit ans traité par Recombinate ${ }^{\circledR}$ pour la réalisation d'une frenectomie.

La traçabilité était assurée par les ordonnances spécifiques des MDS à trois feuillets :

- un feuillet conservé par la pharmacie lors de la délivrance du produit,

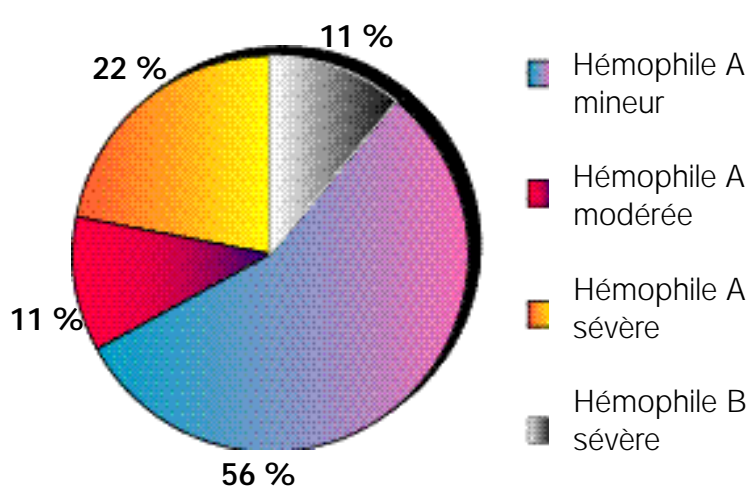

Figure 8 : Types d'hémophilies ayant nécessité l'utilisation de la colle biologique Types of haemophilia needing fibrin glue 
- un feuillet retourné à la pharmacie après administration et identification du praticien,

- le dernier placé dans le dossier du patient.

Les MDS ont permis :

- de diminuer les complications hémorragiques,

- de simplifier la prise en charge des hémophiles,

- de diminuer la durée de l'hospitalisation,

- d'améliorer le confort des patients,

- de diminuer le risque infectieux.

Pour la prise en charge de la maladie Von Willebrand, deux stratégies thérapeutiques sont envisageables [32,38]. La DDAVP ou desmopressine (Minirin ${ }^{\circledR}$ et Octim ${ }^{\circledR}$ spray) qui stimule la libération de tout le facteur Von Willebrand (FvW) présent dans les cellules endothéliales. Elle est le plus souvent efficace pour les types 1 et 2 . Dans le type 3 (forme sévère), la DDAVP est inefficace car les cellules endothéliales ne contiennent pas de FvW mobilisable. L'efficacité est variable car elle dépend de la réponse biologique du patient. Les injections répétées de DDAVP entraînent une diminution progressive du stock cellulaire de FvW, puis deviennent inefficaces (tachyphylaxie). Les MDS d'origine plasmatique sont présentes sous 2 types de formes : le FvW pur (Wilfactin ${ }^{\circledR}$ ) et le FvW associé au FVIII (Wilstart ${ }^{\circledR}$ ). Ce sont des produits substitutifs, efficaces pour toutes les formes cliniques de maladie de Willebrand. Ils sont réservés aux patients pour qui la DDAVP est inefficace ou contre-indiquée. Les deux patients atteints de la maladie de Willebrand ont reçu des injections de $\mathrm{M}$ inirin ${ }^{\circledR}$ associées à une hémostase locale (colle biologique, collagène, suture et compressions) avec une hospitalisation ambulatoires.

Pour les patients traités par l'association d'agents antiagrégants plaquettaires $\mathrm{Kardégic}^{\circledR}$ et Plavix ${ }^{\circledR}[32$, la colle biologique a permis d'obtenir une hémostase locale. Cette as sociation est relativement rare : elle est prescrite à des patients ayant des antécédents d'accidents thromboemboliques très récents (comme les 3 cas traités) ou un risque thrombo-embolique sévère et d'association d'agents antiag régants. Dans ce cas, l'arrêt du traitement ou le relais par une HPBN est contre-indiqué : le risque thrombo-embolique est plus grave que le risque hémorragique. Les patients ont été traités en ambulatoire avec retour au domicile en fin d'après-midi.
La prise en charge des patients à haut risque hémorragique (hémophiles traités par AVK...) nécessite la réalisation d'une hémostase locale. Pour les patients recevant des facteurs de substitution, il y a une consultation initiale conjointe avec l'hématologue pour préciser le cadre de la prise en charge :

- type de MDS utilisé : colle et/ou facteur de substitution,

- évaluation de l'acte à effectuer : difficulté, durée, et suites opératoires éventuelles,

- durée de l'hospitalisation : elle est fonction de l'acte opératoire et de la capacité du patient à réaliser les mesures d'hémostase locale conseillées (compressions intermittentes avec des compresses imbibées d'acide tranexamique - CIA -). Le patient est hospitalisé le jour de l'intervention et reçoit les facteurs de substitution le matin avant I'intervention. $P$ ar ailleurs, la prémédication est établie en fonction de l'acte et du terrain (antibiotiques, anti-inflammatoires stéroïdiens, sédatifs et antalgiques). Le protocole opératoire comporte les étapes suivantes [15, 26, 37, 39]:

- antisepsie buccale et péribuccale,

- anesthésie locale,

- incision ou syndesmotomie,

- luxation et extraction de la dent, ou décollement de la paroi kystique,

- élimination des débris (tissus mous et os) et du tissu de granulation,

- mise en place de la colle biologique,

- mise en place des plaques de collagène (Curaspon ${ }^{\circledR}$ ),

- sutures,

- mise en place de la colle biologique,

- compressions intermittentes avec des compresses imbibées d'acide tranexamique (Exacyl ${ }^{\circledR} 1 \mathrm{~g} .10 \mathrm{ml}^{-1}$ ampoules buvables).

En fin d'après-midi, en l'absence de saignement, le patient quitte I'hôpital. Sinon, un nouveau contrôle est effectué le lendemain matin, car le patient ne quitte l'hôpital qu'en l'absence de saignement. Par la suite, il doit effectuer des compressions avec des compresses imbibées d'acide tranexamique pendant 3 jours (10 minutes toutes

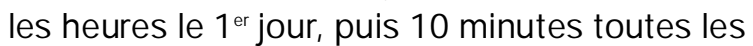
2 heures le $2^{\mathrm{e}}$ jour et toutes les 3 heures le $3^{\mathrm{e}}$ jour). La colle biologique est également utilisée pour les médecine buccale chirurgie buccale VOL. $11, \mathrm{~N}$ 2005 page 155 
médecine buccale chirurgie buccale

L. $11, \mathrm{~N}^{\circ} 3$ 2005

page 156 extractions en terrain irradié, principale cause de l'ostéoradionécrose dont les complications peuvent être sévères (fistule à la peau, fracture spontanée de la mandibule... ). Dans le service, la colle biologique est utilisée systématiquement pour les extractions en terrain irradié. En comblant le fond des alvéoles, elle isole l'os du milieu buccal et surtout de la flore bactérienne. Le facteur XIII présent dans la colle stimule la cicatrisation [40]. De même, l'apport de fibrine favorise le développement de fibroblastes.

Le protocole opératoire est le suivant :

- désinfection buccale et péribuccale,

- incisions et lambeaux de pleine épaisseur,

- extractions et curetage des alvéoles,

- régularisations des crêtes pour permettre une suture bord à bord,

- mise en place de la colle biologique au fond des alvéoles,

- sutures bord à bord.

Huit curetages d'ostéites ont été réalisés en utilisant de la colle biologique suivant le protocole ci-dessus. Elles concernent deux patientes, irradiées chacune pour un carcinome épidermoïde du bord latéral de la langue, chez qui il a été nécessaire de renouveler plusieurs fois la colle.

\section{CONCLUSION}

Les MDS ne sont pas des médicaments comme les autres. Leur origine impose un cadre réglementaire strict dans le but de réduire le risque de transmission d'agents infectieux conventionnels ou non conventionnels. Des moyens existent aujourd'hui pour réduire au minimum le risque viral. Malgré tout le risque zéro n'existe pas, même pour les produits recombinants. La pharmacovigilance exercée sur les MDS assure une traçabilité nécessaire. Elle permet entre autres de

\section{RÉFÉRENCES}

1-Pool JG, Gershgold EJ, Pappenhagen AR. Highpotency antihaemophilic factor concentrate prepared from cryoglobulin precipitate. Nature $1964 ; 203: 312$.

2 - Loi n 93-5 du 4 janvier 1993 relative à la sécurité en matière de transfusion sanguine et de médicament. J ournal Officiel de la République Française du 5 janvier $1993 ; 3: 237-46$. suivre les MDS plasmatiques du donneur au receveur. Le cadre réglementaire du don du sang a été également revu. L'entretien médical, la sélection des donneurs et les tests de dépistage biologiques faits sur chaque don diminuent le risque d'obtenir un lot contaminé. Le risque de don de sang pendant la phase initiale silencieuse de séroconversion virale, devrait être écarté par l'entretien médical. L'essor des méthodes d'élimination/inactivation virales a permis d'assurer la sécurité virale des MDS.

L'amélioration des techniques de production, de fractionnement et de purification du plasma a permis d'obtenir des MDS plasmatiques de haute ou très haute pureté, ce qui conduit à la diminution du nombre d'injections pour obtenir une bonne hémostase.

L'utilisation des MDS en odontologie et en chirurgie buccale concerne essentiellement les actes opératoires à risques hémorragiques, c'est-à-dire principalement les extractions dentaires. Les principaux MDS utilisés dans notre discipline sont les facteurs antihémophiliques, le facteur Von Willebrand et la colle biologique. La colle biologique, associée à la mise en place d'éponge de collagène dans les alvéoles et à des compressions avec compresses imbibées d'acide tranexamique, a permis de simplifier la prise en charge des patients hémophiles, des patients atteints de la maladie Von Willebrand et des patients sous AVK sans modifier l'INR.

Cette évolution de la prise en charge des patients a permis de limiter les complications hémorragiques post-opératoires, de diminuer la durée des hospitalisations et de réduire le risque de contamination virale. Les MDS ont donc contribué à améliorer la qualité des soins pour les patients à haut risque hémorragique.

3 - Décret n 95-566 du 6 mai 1995 relatif à la phamacovigilance exercée sur les médicaments dérivés du sang humain et modifiant le code de santé publique (deuxième partie : Décret en Conseil d'Etat). J ournal Officiel de la République Française du 7 mai 1995 ; 108 : 7373-5. 


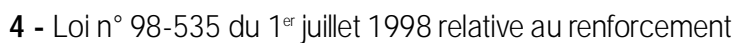
de la veille sanitaire et du contrôle de la sécurité sanitaire des produits destinés à l'homme. J ournal Officiel de la République Française du 5 janvier $1993 ; 151$ : 10056-75.

5 - Directive 89/381/CEE du Parlement Européen et du Conseil de l'U nion Européenne, élargissant le champ d'application des directives 65/65/CEE et 75/319/CEE concernant le rapprochement des dispositions législatives, réglementaires et administratives relatives aux spécialités pharmaceutiques, et prévoyant des dispositions spéciales pour les médicaments dérivés du sang ou du plasma humain. J ournal Officiel de I'Union Européenne du 27 juin 1989 ; L181 : 44-6.

6 - Résolution du Conseil de l'Union Européenne relative à la sécurité transfusionnelle et à l'autosuffisance en sang. J ournal Officiel de l'Union Européenne du 30 juin $1995 ; 164: 216$.

7 - Directive 2002/98/CE du Parlement Européen et du Conseil de l'Union Européenne, établissant des normes de qualité et de sécurité pour la collecte, le contrôle, la transformation, la conservation et la distribution du sang humain et des composants sanguins, et modifiant la directive 2001/83/CE. Journal Officiel de I'Union Européenne du 8 février 2003 ; L033 : 30-40.

8 - Décret n 95-278 du 13 mars 1995 relatif à la pharmacovigilance et modifiant le code de santé publique (deuxième partie : Décret en Conseil d'Etat). J ournal Officiel de la République Française du 14 mars 1995 ; $62: 3935$.

9 - Chamouard V, Lopez I, Stieltjes N. Facteurs antihémophiliques : traitement substitutif de l'hémophilie $A$ et $B$. Publication du Centre d'Information sur le Médicament Hospitalier 2003 ; 24 : 3-81.

10 - C HARLES M. De la fabrication à la délivrance des médicaments dérivés du sang (pp 1-137). Thèse de Diplôme d'Etat de Doctorat en Pharmacie. Université de Reims, 2004.

11 - Service Européen de la Qualité du Médicament. Pharmacopée européenne : addendum 1998 (pp 1591). Conseil de l'Europe, Strasbourg, 1997.

12 - Arrêté du 24 décembre 1997 relatif aux conditions d'utilisation des traitements automatisés des informations dans la pharmacovigilance exercée sur les médicaments dérivés du sang. J ournal Officiel de la République Française du 8 janvier $1998 ; 6$ : 343-4.

13 - Circulaire DGS/DH/99/424 du 19 juillet 1999 relative aux modifications engendrées par le transfert de l'hémovigilance à l'AFSSAPS. Bulletin Officiel 99/29 du 19 au 25 juillet 1999.

14 - TRUong C. Les médicaments dérivés du sang. De leur origine à leur utilisation : moyens de sécurisation et traçabilité (pp 1-220). Thèse de Diplôme d'Etat de Doctorat en Pharmacie. Université de Tours, 2003.

15 - VedRenne A. Obligations du fabricant de médicaments dérivés du sang humain dans la Communauté Européenne et en France ( $p p$ 1-149). Université de Paris Xl, 1999.
16 - AzZI A, Ciappi S, ZakvrZewska K, M orfinı M, Mariani G, MannuCCIPM. Human parvovirus B19 infection in hemophiliacs first infused with two high-purity, virally attenuated factor VIII concentrates. Am J Hematol 1992 ; $39: 228-30$.

17 - Laboratoire Français du Fractionnement et des Biotechnologies Factane ${ }^{\circledR}$ facteur VIII de coagulation humain (pp 1-65). LFB, Courtaboeuf, 2002.

18 - Goudemand J. Hémophilie. Encyclop Médico-Chir, Hématol (pp 18) 13-021-B-10, . Elsevier, Paris, 1997.

19 - HoURDIN-EUdE S. Intérêts du facteur VIla recombinant en odontostomatologie (pp 1-162). Thèse de Diplôme d'Etat de Doctorat en Chirurgie Dentaire. Université de Rennes I, 2003.

20 - HUART J J, B URnOUf T. Le fractionnement plasmatique. STP P harma Pratiques $1992 ; 2: 17-24$.

21 - Paubel P, Sauvageon-Martre H, Wallet P. Les médicaments dérivés du sang (pp 1-390). Arnette, RueilMalmaison, 1999.

22 - J OHNSton A, Adocock W. The use of chromatography to manufacture purer and safer plasma products. Biotechnol Genet Eng Rev $2000 ; 17$ : 37-70.

23 - Lusher J M, ARkin S, AbILdgaARd CF, S ChWARTZ RS. Recombinant factor VIII for the treatment of previously untreated patients with hemophilia A. Safety, efficacy, and development of inhibitors. Kogenate P reviously Untreated Patient Study Group. N Engl J Med. 1993; $328: 453-9$.

24 - Bray GL, Gomperts ED, Courter $\mathrm{S}, \mathrm{G}$ ruppo R, Gordon EM, Manco-J ohnson M, Shapiro A, Scheibel E, White G, LEE M. A multic enter study of recombinant factor VIII (recombinate): safety, efficacy, and inhibitor risk in previously untreated patients with hemophilia $A$. The Recombinate Study Group. Blood 1994 ; 83 : 242835.

25 - NÉGRIER C. Le traitement de l'hémophilie: des dérivés du plasma à la thérapie génique. Hématologie 1996 ; $2: 17-27$

26 - NÉGRIER C. The current therapeutic bases for hemophilia. Transfus Clin Biol $2000 ; 7: 257-8$.

27 - VeHAR GA.P roduction of recombinant coagulation proteins. Ann N Y Acad Sci $1987 ; 509$ : 82-8.

28 - Samson J, de Moerloose P, Fiore-Donno G. Les troubles de l'hémostase en médecine dentaire. Rev Mens Suisse Odonto-stomatol $1987 ; 97: 1398-402$

29 - Bernard J P, Lombardi T, SAmson J. Use of a fibrin sealant for dental extractions in irradied jaws (pp 172-175). In Fibrin Sealant in Operative Medicine, Plastic Surgery, Maillofacial and Dental Surgery, G. Schlag \& H Redl Eds, Springer-Verlag, Berlin, 1994

30 - Lettre du comité du médicament. Centre Hospitalier Universitaire de Rennes $2003 ; 73: 1-35$.

31 - Lettre du comité du médicament. Centre Hospitalier Universitaire de Rennes $2003 ; 74: 1$-15.

32 - Breton-Gorius J, Reyes F, Rochant H, Rosa J, VERnANT J P. L'hématologie de Bernard Dreyfus (pp 11474). Flammarion Médecine/Sciences, Paris, 1992. médecin€

buccale chirurgie buccale VOL. 11, N 2005 page 157 
33 - Hylek EM, Go AS, Chang Y, J ensvold NG, Henault Le, SELBY J V, SINGER DE.Effect of intensity of oral anticoagulation on stroke severity and mortality in atrial fibrillation. N EnglJ Med $2003 ; 349$ : 1019-26.

34 - Auerswald G, Spranger T, Brackmann HH. The role of plasma-derived factor VIII/von Willebrand factor concentrates in the treatment of hemophilia A patients. Haematologica/J ournal of Hematology $2003 ; 88$ (suppl 9) : 21-25.

35 - DJ ULBEGOVIC B, GoldSMITH GH. Guidelines for management of hemophilia A and B. Blood $1995 ; 85: 598-9$.

36 - Lloyd J Ones M, Wight J , Paisley S, KNight C. Control of bleeding in patients with haemophilia $A$ with inhibitors: a systematic review. Haemophilia $2003 ; 9$ : 464520.

37 - Rakocz M, Mazar A, Varon D, Spierer S, Blinder D, MarTinowitz U. Dental extractions in patients with bleeding disorders. The use of fibrin glue. Oral Surg Oral Med Oral Pathol Oral Radiol Endod 1993 ; 75 : 280-2.
38 - Lethagen S, Harris AS, SJ ORIN E, Nilsson IM.Intranasal and intravenous administration of desmopressin: effect on F VIII/vWF, pharmacokinetics and reproducibility. Thromb Haemost 1987 ; 58 : 1033-6.

39 - Frachon X, Pommereuil M, Berthier AM, Lejeune S, Hourdin-Eude S, Quero J, Meziere X, De Mello G, GARNIER J . Management options for dental extraction in hemophiliacs: a study of 55 extractions (2000-2002). Oral Surg Oral Med Oral Pathol Oral Radiol Endod $2005 ; 99: 270-5$.

40 - Matras $H$. The use of fibrin sealant in oral and maxillofacial surgery. J Oral Maxillofac Surg 1982 ; 40 : 61722. médecine

page 158 